\title{
From imitation to implementation: How two- and three-year-old children learn to enforce social norms
}

\author{
Susanne Hardecker* and Michael Tomasello \\ Max Planck Institute for Evolutionary Anthropology, Leipzig, Germany
}

\begin{abstract}
Young children enforce social norms from early on, but little research has examined how this enforcement behaviour emerges. This study investigated whether observing an adult's norm enforcement influences children's own enforcement of that norm compared with observing an action demonstration without enforcement. Additionally, children experienced enforcement either following their own (second-party) or a third-party's transgression $(N=120)$. Results revealed that observing enforcement increased twoand three-year-old children's protest against the sanctioned action regardless of secondor third-party context. However, only three-year-olds generalized their enforcement to a novel action not matching the norm, whereas two-year-olds only protested against the previously sanctioned action. Importantly, without any enforcement demonstration, twoyear-olds rarely protested at all while three-year-olds did so quite frequently. Thus, providing an opportunity to imitate enforcement seems to give rise to enforcement behaviour in two-year-olds while three-year-olds already understand normative implications following a variety of cues and even apply norm enforcement without any demonstration of how to do it.
\end{abstract}

\section{Statement of contribution}

What is already known on this subject?

- Children conform to social norms from early in development.

- Young children from 2 to 3 years of age also enforce social norms on third parties.

What does this study add?

- Observing enforcement by an adult increases two- and three-year-olds' protest against the sanctioned action.

- It does not matter whether children experienced enforcement on their own or a third party's action.

- Three-, but not two-year-olds, generalize their enforcement to novel actions that do not match the norm.

Children grow up in a society that works according to specific social norms. From early on, parents enforce these rules of conduct on their children, who start conforming from around their first birthdays (Dahl \& Campos, 2013; Gralinski \& Kopp, 1993; Smetana, Kochanska, \& Chuang, 2000). This early compliance to norms is mostly driven by a prudential respect for the authority of adults (Piaget, 1932).

*Correspondence should be addressed to Susanne Hardecker, Max Planck Institute for Evolutionary Anthropology, Deutscher Platz 6, Leipzig 04 103, Germany (email: susanne_hardecker@eva.mpg.de). 
However, a relatively recent discovery is that young children from around 2 to 3 years of age will actually enforce social norms on others themselves, even in thirdparty contexts in which they are not affected at all. In this case, there are no obvious prudential motives for children to enforce social norms, and so the question arises how young children come to show this behaviour at all.

Young children's norm enforcement occurs across a wide range of situations (Rossano, Rakoczy, \& Tomasello, 2011; Wyman, Rakoczy, \& Tomasello, 2009). For example, Vaish, Missana, and Tomasello (2011) have shown that when three-year-old children observe someone committing a moral transgression by destroying the property of an absent actor, they intervene and protest against this harmful act on behalf of the victim. Even more surprisingly, however, such intervention and protest also occur after the transgression of merely conventional norms that affect no one at all. For example, when young children are taught how to play a novel game and they observe a puppet actor playing this game in the wrong way, they also protest against this non-canonical action (Rakoczy, Warneken, \& Tomasello, 2008). These cases are most remarkable as they involve an arbitrary and solitary game activity, that is, the action performed does not have an instrumental (e.g., procuring some resource) or moral purpose (e.g., helping someone) but simply constitutes the way 'we' play the game. Studies from diverse cultural settings are now accumulating, revealing that young children in the United States (Casler, Terziyan, \& Greene, 2009), Germany (Schmidt, Rakoczy, \& Tomasello, 2012), Sweden (Kenward, 2012; Kenward, Karlsson, \& Persson, 2011), and Turkey (Tuncgenc, Hohenberger, \& Rakoczy, 2015) begin to enforce norms at around the same age in ontogeny.

But how does the behaviour of norm enforcement emerge in children in the first place? One possibility is that for moral transgressions, children have a sympathetic concern for someone harmed and thus attempt to help the victim by preventing that harm (Vaish, Carpenter, \& Tomasello, 2009). Regarding transgressions of conventional game rules, they might react out of a sense of integrity of the game and an implicit sense that for things to work, rules must be followed. But another possibility is that when children are enforcing norms, they are imitating adults' enforcement of norms. Soon after their first birthdays, infants can imitate instrumental actions in a systematic way by taking into account the rationality of the action as well as the actor's underlying intentions (Carpenter, Akhtar, \& Tomasello, 1998; Gergely, Bekkering, \& Kiraly, 2002; Meltzoff, 1995; Schwier, Van Maanen, Carpenter, \& Tomasello, 2006). Thus, at 2-3 years of age, children might imitate adult norm enforcement and this could be underlain by different degrees of comprehension of the action. Almost certainly, three-year-old children are not only mimicking surface behaviour as they have been found to enforce norms in a variety of novel and arbitrary play situations in which they had not observed any enforcement previously (Schmidt \& Tomasello, 2012). Particularly remarkable in this regard is the finding of the emergence of uncued norm enforcement in three-yearolds who also enforce an arbitrary action after only observing an adult performing that action intentionally without explicit teaching, that is, without the adult explicitly marking the behaviour as a rule (Schmidt, Rakoczy, \& Tomasello, 2011). This finding suggests that three-year-olds readily infer normativity from intentionally performed actions without needing any direct explicit teaching or enforcement. However, these previous studies do not address whether and how social learning mechanisms might give rise to children's initial enforcement behaviour of conventional rules in the first place and which learning contexts shape children's 
tendencies to enforce rules. Therefore, this study aimed to investigate how social learning opportunities of enforcement behaviour influence two- and three-year-old children's own inclinations to enforce norms on others.

If we assume that a somewhat flexible form of social learning and imitation plays some role in children's norm enforcement, this could still occur in two different ways. First, it might be that children enforce social norms on third parties because they have seen adults enforce social norms on third parties (e.g., their siblings). Second, it might be that they enforce norms on third parties based on their own experience with having norms directly enforced on them (second-party) and then they generalize this behaviour to third-party contexts. Previous research has addressed the question of second- versus third-party imitation mostly in the area of language acquisition. For example, Akhtar and colleagues have found that from around 18 months of age, children learn novel words for objects as efficiently from overhearing a third-party as from a second-party interaction addressed directly towards them (Akhtar, 2005; Akhtar, Jipson, \& Callanan, 2001; Floor \& Akhtar, 2006). In terms of instrumental actions, Shneidman, Todd, and Woodward (2014) found that 18-month-old infants imitated more often in second-party than in third-party modelling contexts; 25 -month-olds imitated equally in both contexts. With regard to norm enforcement in particular, there are no empirical data to our knowledge. But Royzman, Leeman, and Baron (2009) make the interesting suggestion that the natural default perspective through which one becomes privy to the prevailing social norms [...] would be that of a perpetrator' (p. 164). Thus, we might hypothesize that by transgressing a norm themselves and being chastised by an adult, children become more aware of the existence of the norm and the subsequent norm enforcement.

In the current study, we gave two- and three-year-old children the opportunity to enforce a social norm on a third party who broke a conventional game rule. Our question was whether social learning influences the emergence of enforcement behaviour in young children. Thus, we investigated whether children who had experienced enforcement of the game rule previously would be more likely subsequently to enforce the rule on a violator as compared to children who had experienced no enforcement of the rule but only a positive demonstration of the action (as in Schmidt et al., 2011). Moreover, given some role of imitation, we asked whether it matters if children experience adult norm enforcement on their own rule violation or on a third party's rule violation. Finally, we were interested in whether children would enforce the previously sanctioned action to the same degree as a novel action that has not been demonstrated or sanctioned before but still does not match the norm. Answers to these questions will help us to address the more fundamental question of why children enforce social norms whose violation does not affect them directly.

\section{Method}

\section{Participants}

Two- and three-year-old children from mixed socio-economic backgrounds participated in this study and were recruited in their respective day care centres (age range: $2.3-2.7$, $M=2.5$ and 3.3-3.7, $M=3.6$ ). Parental consent was given prior to testing. Overall, 120 children were included into the final sample (60 two-year-olds, 60 three-year-olds; 60 female). Ten additional children were tested but had to be excluded from the final sample due to experimenter error $(n=5)$, inattentiveness during test phase $(n=4)$, and technical equipment failure $(n=1)$. 


\section{Materials}

Overall, five animal hand puppets were used in the study: frog, bear, rabbit, dog, and cow. The frog was used as an introductory puppet to familiarize children with the situation and the presence of an experimenter playing the puppets. The rabbit, dog, and cow puppet were used during the warm-up and during test phase as the test puppets, and the bear puppet was used during the manipulation phase. For the warm-up, a stacking tower with coloured wooden discs and a puzzle were used. The objects that were used for the test consisted of a red wooden block with a hole and a wooden stick (see Figure 1).

\section{Design and procedure}

The study design comprised three between-subjects conditions named as follows: secondparty enforcement (2P), third-party enforcement (3P), and the no enforcement control (control). Twenty two-year-old and 20 three-year-old children (half of them female) were randomly assigned to each condition.

Two experimenters conducted the study; experimenter 1 (E1) was the instructor and experimenter 2 (E2) played all of the hand puppets. In the beginning of the study, both experimenters picked up the child from her kindergarten group whereby E2 played the frog puppet to introduce the child to the play situation. When the child felt comfortable, the experimenters took the child to the testing room in which a table including three chairs was set up and the three test puppets (rabbit, dog, and cow) were already put on stands on the table. The child was invited to take a seat next to E1 and opposite to the puppets (see Figure 2 for exemplary pictures of the setup). The frog puppet announced right away that she was tired and went to sleep whereupon E1 introduced each test puppet to the child (fixed order: dog, cow, rabbit). Two warm-up games followed during which the child and the puppets played together and took turns in stacking a tower with coloured wooden discs and in completing a puzzle together. Each of the puppets committed one instrumental mistake during these games while E1 was turned away from the scene (i.e., failing to stack a disc and putting the puzzle piece upside down or sideways). These mistakes gave the child the opportunity to correct the puppets which was important in order to clearly mark the puppets as non-authoritarian figures that act child-like and make mistakes and to allow the child to overcome shyness when interacting with the puppets. After these warm-ups, all three puppets declared that they were tired
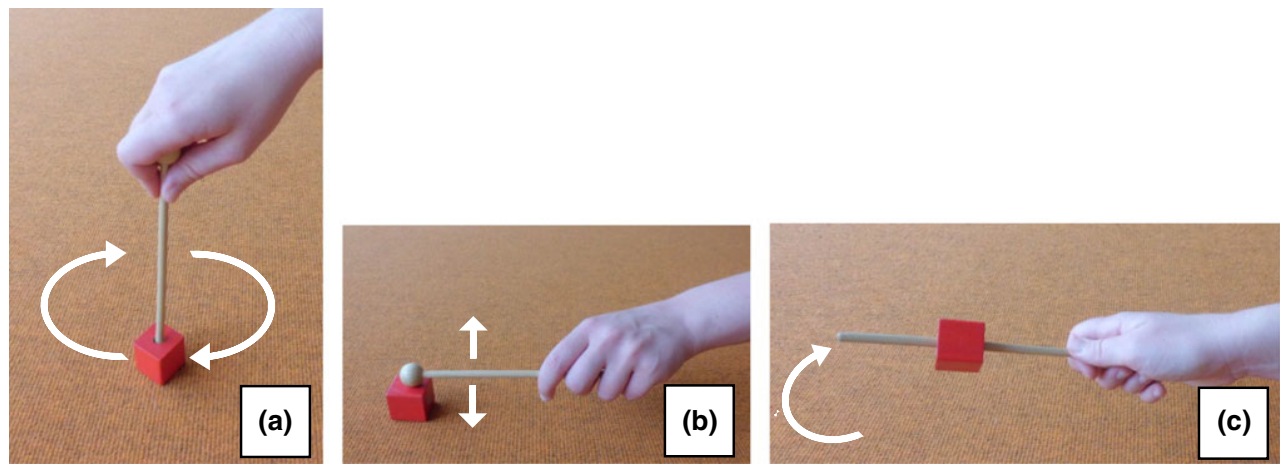

Figure I. Actions performed with test objects: (a) moving the block in circles using the stick; (b) knocking on the block with the stick; (c) threading the block on the stick. 

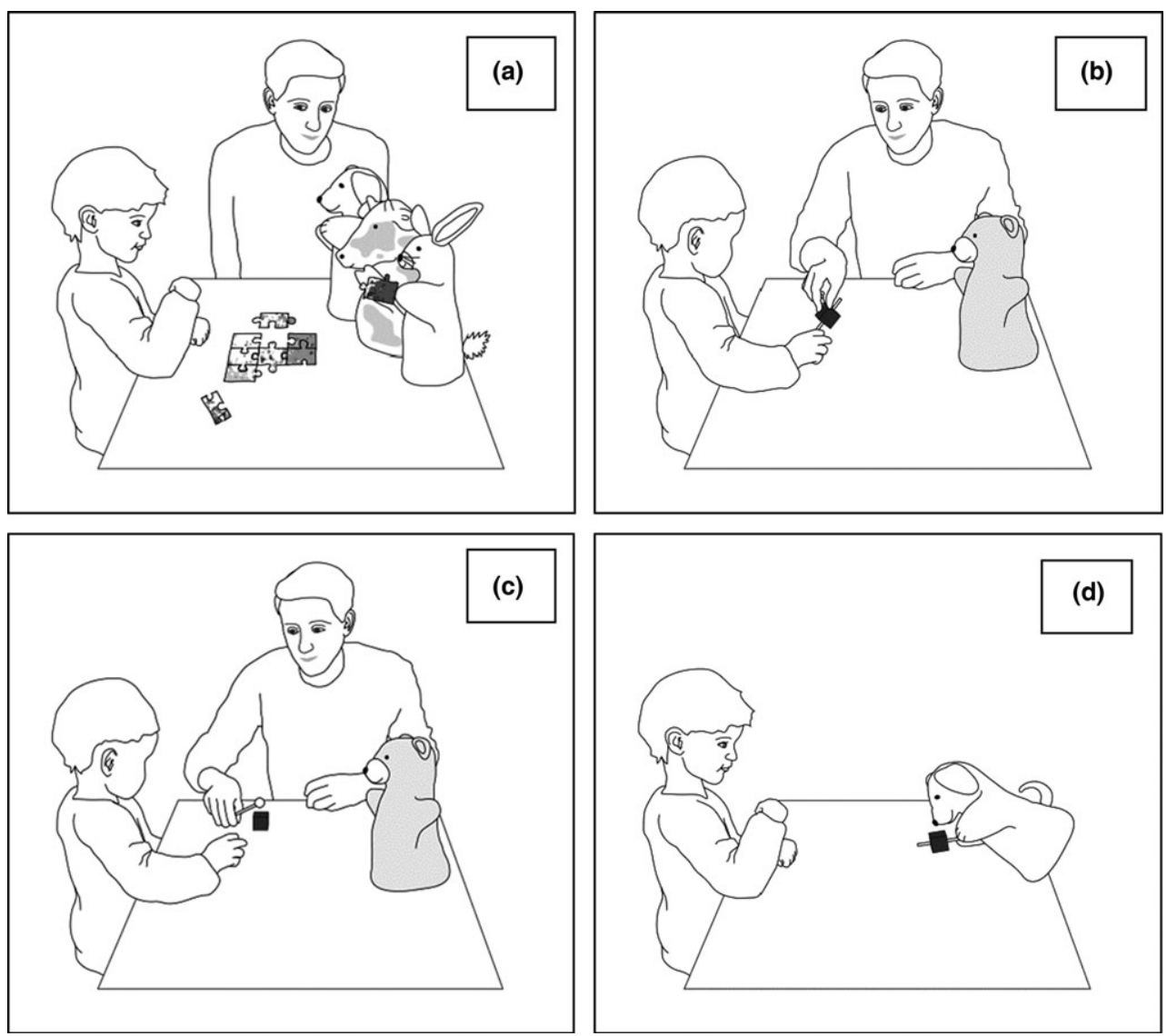

Figure 2. Details of the procedure: (a) Warm-up with the test puppets; (b, c) manipulation phase (2P): (b) El takes test objects from the child who has performed a spontaneous action, declares it as 'wrong' and (c) enforces the 'correct' action directly towards the child; (d) test phase: one puppet performs the wrong action.

and went to sleep (i.e., were put under the table by E2) whereupon the bear puppet appeared which marked the beginning of the manipulation phase.

\section{Manipulation phase - 2P condition}

E1 put the objects for the test game on the table and told the child: 'I have brought another game and you can play with this now'. He then handed the objects to the child and waited for the child to perform any identifiable playing action with the objects. As soon as the child did so, the experimenter took the game from the child saying:

No, [name of child]! That's not how it goes at all. This [E1 imitates the wrong action] is wrong. One mustn't do it like this. It actually goes like this [E1 demonstrates the correct action]. This is how the game goes. One must do it like this [E1 demonstrates the correct action again].

Thus, any playing action the child performed was declared as 'wrong' and E1 offered an alternative 'correct' playing action. Even though children differed regarding which 
action they performed spontaneously, most of the children either knocked on the block or threaded the stick through the block (see Figure 1b, c) and E1 would then choose an alternative 'correct' action from the three main possibilities depicted in Figure 1.

\section{Manipulation phase $-3 P$ condition}

This condition proceeded exactly as the $2 \mathrm{P}$ condition, except that $\mathrm{E} 1$ hands the test objects to the bear instead of the child. Then the bear performs a play action and E1 declares this action as 'wrong' and enforces the 'correct' way on the bear instead of the child using the exact wording as in the $2 \mathrm{P}$ condition but this time directed at the bear.

\section{Manipulation phase - Control condition}

$\mathrm{E} 1$ hands the test objects to the bear puppet again and then turns away from the scene. The bear says: 'Oh, what is this? I don't know this game at all. I will try it now'. The bear performs a playing action twice (which is the 'wrong' action as he is clearly ignorant of the game) and puts down the objects on the table whereupon E1 turns back to the table and says: 'Oh, I know this game! This is a great game! I am going to play it now'. E1 takes the objects and performs the 'correct' action twice.

In each of the conditions, the child saw the wrong and the correct action performed twice but was either exposed to a direct enforcement on herself or on a third party or experienced no enforcement at all but just observed intentional actions performed by an ignorant and a knowledgeable agent.

As the specific action children performed in the $2 \mathrm{P}$ condition varied between children, the $3 \mathrm{P}$ and the control conditions were yoked to the $2 \mathrm{P}$ condition. Thus, for each action combination of what was 'wrong' and 'correct' for a child in the $2 \mathrm{P}$ condition, another child in the $3 \mathrm{P}$ condition as well as in the control condition received the exact same action combination, that is, in $3 \mathrm{P}$ the bear would then perform the wrong action that another test child in $2 \mathrm{P}$ had performed and E1 would teach the bear the correct action that was also taught to the child in 2P (and the same for the control condition). Therefore, every child was matched with two other children in the other conditions regarding which actions were wrong and correct.

After this manipulation phase, E1 left the room to take care of something outside and the test phase started. One after another, the test puppets appeared in front of the child to play with the objects and each puppet performed one specific play action three times in a row to give the child enough time to respond to the action. These actions were either the wrong, the correct, or a novel way of playing with the objects according to what the child had learnt during the manipulation phase. The order of the puppets (rabbit, dog, cow) was counterbalanced, and the order of the actions which they performed (wrong, correct, novel) could proceed in two ways which were also counterbalanced: (1) wrong action, correct action, novel action or (2) novel action, correct action, wrong action. The second puppet always performed the correct playing action because this served as an in-between buffer trial to ensure children were not simply put in a protesting mood and protested indiscriminately against all the performed actions but recognized the correct action.

\section{Coding and reliability}

The whole procedure was recorded on video from which children's responses were coded afterwards. Our main interest was children's enforcement behaviour, and 
therefore, we coded whether children protested against the wrong, correct, and novel action performed by the test puppets. We also coded whether children's protest included normative language or was imperative and implicit (cf. Schmidt et al., 2012). Normative language includes words such as must, ought, should, right/correct, and wrong and regarding objects as referents words such as go, belong (e.g., 'This doesn't go there', 'It doesn't belong there like this'; see Appendix for the specific coding categories). A second independent coder blind to conditions coded 25\% of the data and Cohen's kappa indicated a good inter-rater reliability for the coding of responses as normative, imperative, or no protest $(\kappa=.85)$.

\section{Results}

Descriptive results are illustrated in Figure 3. To investigate the effect of experiencing enforcement on children's own tendency to enforce a game rule, we fitted a generalized linear mixed model (GLMM) in R (version 3.2.2, R-Core-Team, 2015) with a binomial error structure and a logit link function using the package lme 4 (Bates, Maechler, Bolker, \& Walker, 2014).

\section{2-year-olds}

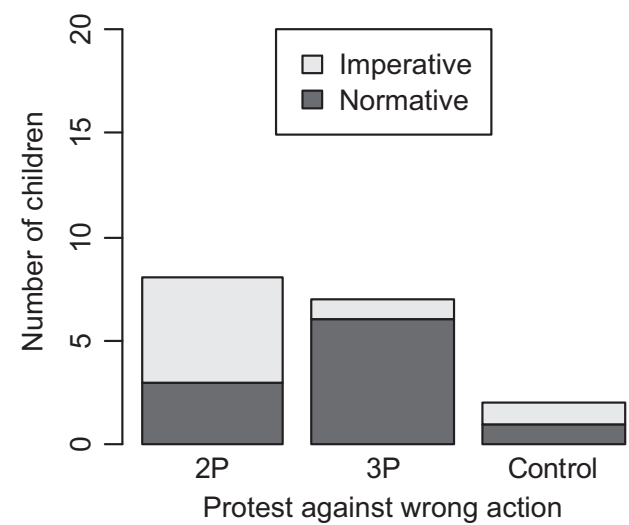

2-year-olds

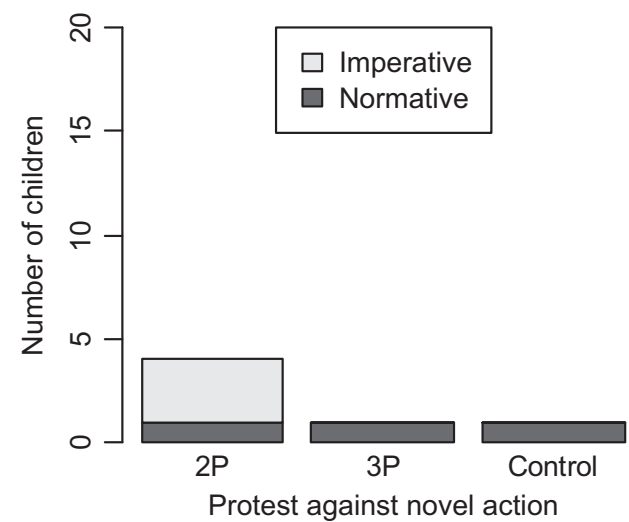

\section{3-year-olds}

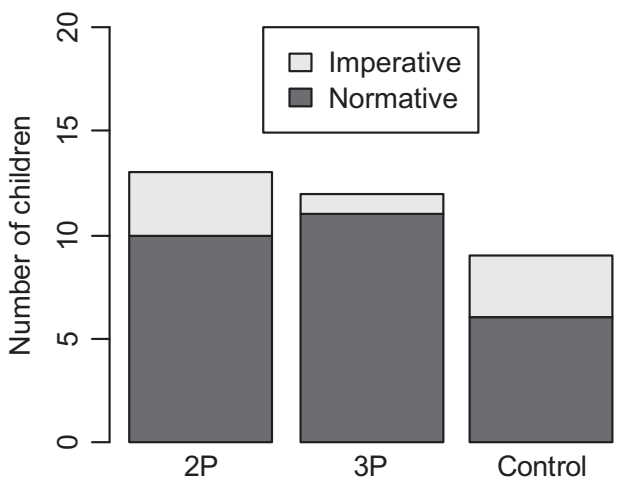

Protest against wrong action

\section{3-year-olds}

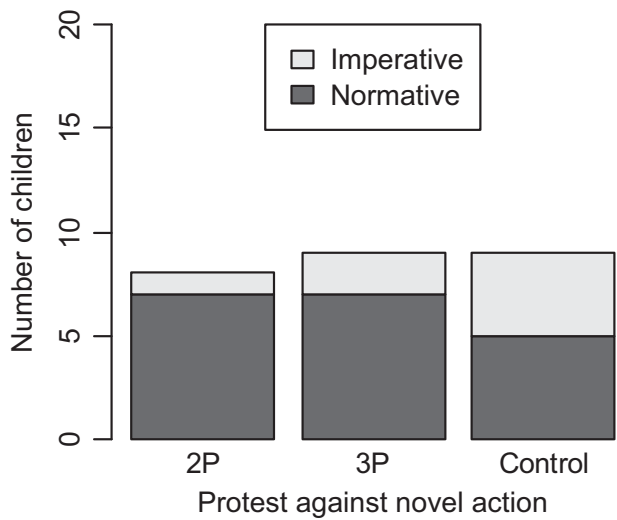

Figure 3. Number of children protesting against the wrong or novel action across conditions and ages. 
Table I. Generalized linear mixed model

\begin{tabular}{lrrrr}
\hline & Estimate $^{\mathrm{a}}$ & $S E$ & $\chi^{2 \mathrm{~b}}$ & $p$ \\
\hline Intercept & -13.201 & 3.245 & & \\
Condition (2P vs. control) & -0.313 & 2.713 & & \\
Condition (3P vs. control) & -0.156 & $2.85 \mathrm{I}$ & & \\
Action type (wrong vs. novel) & 10.665 & 3.155 & & \\
Age (3 vs. 2) & 2.654 & 2.714 & & $<.001$ \\
Sex (female vs. male) & 0.766 & 0.468 & & \\
Condition $\times$ Action type (2P, wrong) & 1.782 & 2.733 & 10.729 & \\
Condition $\times$ Action type (3P, wrong) & 1.356 & 2.866 & & \\
Action type $\times$ Age (wrong, 3) & -1.014 & 2.729 & 20.295 & $<.001$ \\
\hline
\end{tabular}

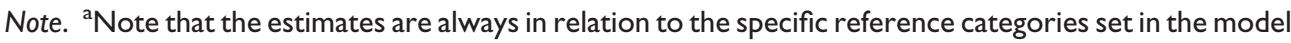
which are in this case condition $=$ control, action type $=$ novel, age $=$ two-year-olds, sex $=$ male; for example, the intercept refers to the estimate for male two-year-old children in the control condition protesting against the novel action.

${ }^{b}$ Chi-square values are only indicated for the significant interaction effects and not for the main effects involved in these interactions as their interpretation would not be meaningful.

The model included condition (2P, 3P, control), age group (two- and three-year-olds), and action type (wrong action, novel action) as our fixed effects of interest as well as all of their interactions. Children's protest was the dependent binary measure. As each child provided one data point for protest against the wrong action and one for the novel action, the random effect of child as well as the random slope of the effect of action type within the random effect of child was also included into the model. To control for the effects of sex, experimenter, order of the test puppets, and order of the test actions (wrong or novel action first), these were also included into the model as fixed effects.

In a first step, the full model as described above was compared to a null model which did not include the fixed effects of interest (condition, age group, action type) but all other effects, and this revealed a significant effect of these variables $\left(\chi^{2}=53.86, d f=11\right.$, $p<.001$ ). In a second step, the fit of the full model was compared to a reduced model not including the three-way interaction of the fixed effects which revealed that the three-way interaction was not significant $\left(\chi^{2}=0.00, d f=2, p=1\right)$. Finally, the fit of further reduced models lacking individual two-way interactions was tested and revealed a significant interaction between condition and action type $\left(\chi^{2}=10.73, d f=2, p<.01\right)$ such that the conditions including an enforcement demonstration (2P and $3 \mathrm{P}$ ) led to higher protesting compared with the control condition, but this effect was only true for protest against the wrong action whereas there was no effect of condition on the protest against a novel action. Furthermore, there was a significant interaction of age and action type $\left(\chi^{2}=20.30\right.$, $d f=1, p<.001$ ) such that three-year-olds protested against the wrong as well as the novel action but two-year-olds protested mostly against the wrong and only rarely against the novel action. Further information on the models can be found in Table 1 but note that for the sake of clarity not all of the fixed effects that were controlled for are displayed.

\section{Discussion}

The present study investigated whether social learning via imitation prompts the emergence of norm enforcement behaviour in young children and, if so, whether 
experiencing enforcement on one's own norm transgressions affects children differently than observing enforcement on a third party's transgression. Overall, we found that prior demonstration of enforcement by an adult increased children's own enforcement of the previously sanctioned action regardless of whether children had experienced this enforcement directly in a second-party context or indirectly in a third-party context. However, in a control condition without any enforcement but merely the demonstration of game actions by a knowledgeable and an unknowledgeable actor, children protested significantly less against this wrong action. Importantly, this effect of enforcement demonstration only applied to children's enforcement of the previously sanctioned action but not to a novel action that had not been demonstrated before. When children saw a new action that did not match the norm, having experienced previous enforcement by an adult did not influence children's protest against that unknown novel action. This suggests that the social learning effect of an enforcement demonstration only affects children's protest on the specifically modelled action and does not translate into a more general implementation of that norm defined more broadly.

Interestingly, even in the control condition almost half of the three-year-old children enforced the norm and protested against the sanctioned as well as the novel action without having seen enforcement in this particular context previously, whereas only one and two of the 20 two-year-olds did so. Children's enforcement of the novel action in the control condition partially replicates previous findings by Schmidt et al. (2011) who found that three-year-olds enforce norms after only seeing an intentional action by a knowledgeable actor without previous explicit normative teaching of that action. Our results now extend these findings and show that this does not apply to two-year-old children. While three-year-olds seem to infer normativity from a variety of cues (explicit teaching, enforcement demonstration, intentional action) and conclude that any mismatch to the demonstrated behaviour violates this norm, two-year-olds need the explicit enforcement demonstration of a wrong action to draw this conclusion - and then do so only for this specific action. Therefore, the emergence of enforcement behaviour in two-year-olds relies much more on social learning than it does for three-year-olds. Moreover, although two-year-olds are able to learn via social learning that a specific action violates a norm and so is sanctionable, three-year-olds additionally infer that there is a more general normative expectation for conformity to the norm, and thus, they also sanction a novel action. This age difference in children's understanding of social norms corresponds to other research and theory that portrays children's normativity in toddlerhood as within the confines of a second-personal morality which relies on individual assessments of others' behaviour based on personal relationships and social emotions. Only around 3 years of age are children then able to recognize that there is an objectivity to norms beyond the realm of second-personal relationships (Tomasello, 2016; Tomasello \& Vaish, 2013).

In a different study by Butler, Schmidt, Burgel, and Tomasello (2015), it has been shown that three-year-old children's normative inferences are stronger (i.e., they upheld their protest for longer) after a pedagogical demonstration compared with a mere observation. With regard to the current study, an alternative explanation of the effects might also be that experiencing enforcement either in a second- or third-party context increases the strength of children's normative inference. This effect of enforcement demonstration might particularly spur two-year-olds' enforcement as they might be less certain of the normative implications than three-year-olds, and thus, the explicit enforcement by an adult might increase their confidence that the sanctioned action is wrong. Thus, potentially two-year-olds did not simply imitate adults' enforcement of the wrong action but were merely more certain after having seen an enforcement 
demonstration. Our study cannot rule out this alternative possibility, and future investigations are needed to disentangle these effects by testing additional conditions that might increase children's confidence without giving them the opportunity to imitate enforcement.

These findings have interesting implications for the study of norm enforcement in different cultural settings. A recent debate has revolved around teaching in cross-cultural contexts, which can take many different forms such as explicit verbal instruction or the mere provision of learning opportunities (Kline, 2014; Rogoff, Mistry, Goncu, \& Mosier, 1993). In this same way, we might suspect that norm enforcement by explicit protest is only one of many possible different ways in which children are familiarized with the prevailing social norms of their culture. Thus, in some cultural contexts, enforcement behaviour might not typically be seen in children's upbringing and it is an open empirical question whether children in these cultures enforce norms by protesting at all. As imitating enforcement seems fundamental for two-year-olds' acquisition of enforcement behaviour, a cultural context in which there is no model for norm enforcement might also not produce this kind of norm enforcement in children at all. Rather, we might suspect that there are different routes to how children acquire normative information about their culture and also how they acquire appropriate behavioural responses to norm transgressions.

Interestingly, children behaved similarly in second- and third-party enforcement contexts and took both occasions as equally informative for their own construal and responses to the situation. While this is in line with previous work showing that children learn conventional and instrumental actions equally well in both contexts (e.g., Nielsen, Moore, \& Mohamedally, 2012), we assumed that younger children might be more inclined to imitate enforcement after they have personally experienced it following their own norm violation (also based on the suggestion by Royzman et al., 2009, that normative learning might be especially spurred by being the transgressor oneself). It might be the case that our manipulation was not strong enough to make a difference for children's experience of the enforcement. As the experimental situation was very interactive and enforcement in second- as well as third-party contexts took place on a table at which the experimenter, the puppet, and the child were seated, children might still have felt very involved into the third-party situation as they sat directly opposite to the transgressor. Hence, observing enforcement more incidentally might reduce children's enforcement rates in the third-party context, but this question would need to be addressed by future research.

Overall, the present study suggests a developmental trajectory of children's norm enforcement that emerges in two-year-old children based on their capacities to imitate the enforcement behaviour of an adult in the same situation in which they observed it, but they do not yet generalize enforcement to novel actions and they rarely enforce norms after mere demonstrations of intentional actions. By 3 years of age, however, children are reliable norm enforcers who can flexibly implement their understanding of normativity and generalize enforcement behaviour following various kinds of demonstrations.

\section{Acknowledgements}

Our gratitude goes to Jana Jurkat for her great assistance and coordination of this project, to Benjamin Schmid and Julia Lang for their valuable and competent help in collecting the data, and to Marike Schreiber for the skilful illustrations. We would also like to thank all the parents, teachers, and children for their participation in this study. 


\section{References}

Akhtar, N. (2005). The robustness of learning through overhearing. Developmental Science, 8, 199209. doi:10.1111/j.1467-7687.2005.00406.x

Akhtar, N., Jipson, J., \& Callanan, M. A. (2001). Learning words through overhearing. Child Development, 72, 416-430. doi:10.1111/1467-8624.00287

Bates, D., Maechler, M., Bolker, B., \& Walker, S. (2014). lme4: Linear mixed-effects models using Eigen and S4 (Version R package version 1.1-5). Retrieved from http://CRAN.R-project.org/ package $=1 \mathrm{me} 4$

Butler, L. P., Schmidt, M. F. H., Burgel, J., \& Tomasello, M. (2015). Young children use pedagogical cues to modulate the strength of normative inferences. British Journal of Developmental Psychology, 33(4), 476-488. doi:10.1111/bjdp.12108

Carpenter, M., Akhtar, N., \& Tomasello, M. (1998). Fourteen- through 18-month-old infants differentially imitate intentional and accidental actions. Infant Behavior \& Development, 21, 315-330. doi:10.1016/S0163-6383\%2898\%2990009-1

Casler, K., Terziyan, T., \& Greene, K. (2009). Toddlers view artifact function normatively. Cognitive Development, 24, 240-247. doi:10.1016/j.cogdev.2009.03.005

Dahl, A., \& Campos, J. J. (2013). Domain differences in early social interactions. Child Development, 84, 817-825. doi:10.1111/cdev.12002

Floor, P., \& Akhtar, N. (2006). Can 18-month-old infants learn words by listening in on conversations? Infancy, 9, 327-339. doi:10.1207/s15327078in0903_4

Gergely, G., Bekkering, H., \& Kiraly, I. (2002). Rational imitation in preverbal infants. Nature, 415, 755. doi: $10.1038 / 415755 a$

Gralinski, J. H., \& Kopp, C. B. (1993). Everyday rules for behavior: Mothers' requests to young children. Developmental Psychology, 29, 573-584. doi:10.1037/0012-1649.29.3.573

Kenward, B. (2012). Over-imitating preschoolers believe unnecessary actions are normative and enforce their performance by a third party. Journal of Experimental Child Psychology, 112, 195-207. doi:10.1016/j.jecp.2012.02.006

Kenward, B., Karlsson, M., \& Persson, J. (2011). Over-imitation is better explained by norm learning than by distorted causal learning. Proceedings of the Royal Society B, 278, 1239-1246. doi: $10.1098 / \mathrm{rspb} .2010 .1399$

Kline, M. A. (2014). How to learn about teaching: An evolutionary framework for the study of teaching behavior in humans and other animals. Behavioral and Brain Sciences, 38, e31. doi: $10.1017 /$ S0140525X14000090

Meltzoff, A. N. (1995). Understanding the intentions of others: Re-enactment of intended acts by 18month-old children. Developmental Psychology, 31, 838-850. doi:10.1037/0012-1649.31. 5.838

Nielsen, M., Moore, C., \& Mohamedally, J. (2012). Young children overimitate in third-party contexts. Journal of Experimental Child Psychology, 112(1), 73-83. doi:10.1016/j.jecp. 2012.01.001

Piaget, J. (1932). The moral judgment of the child. London, UK: Routledge \& Kegan Paul.

Rakoczy, H., Warneken, F., \& Tomasello, M. (2008). The sources of normativity: Young children's awareness of the normative structure of games. Developmental Psychology, 44, 875-881. doi:10.1037/0012-1649.44.3.875

R-Core-Team (2015). R: A language and environment for statistical computing. Vienna, Austria: Author. Retrieved from https://www.R-project.org/

Rogoff, B., Mistry, J., Goncu, A., \& Mosier, C. (1993). Guided participation in cultural activity by toddlers and caregivers. Monographs of the Society for Research in Child Development, 58(8), 1-179. doi:10.2307/1166109

Rossano, F., Rakoczy, H., \& Tomasello, M. (2011). Young children's understanding of violations of property rights. Cognition, 121, 219-227. doi:10.1016/j.cognition.2011.06.007

Royzman, E. B., Leeman, R. F., \& Baron, J. (2009). Unsentimental ethics: Towards a content-specific account of the moral-conventional distinction. Cognition, 112(1), 159-174. doi:10.1016/ j.cognition.2009.04.004 
Schmidt, M. F. H., Rakoczy, H., \& Tomasello, M. (2011). Young children attribute normativity to novel actions without pedagogy or normative language. Developmental Science, 14, 530-539. doi:10.1111/j.1467-7687.2010.01000.x

Schmidt, M. F. H., Rakoczy, H., \& Tomasello, M. (2012). Young children enforce social norms selectively depending on the violator's group affiliation. Cognition, 124, 325-333. doi:10.1016/ j.cognition.2012.06.004

Schmidt, M. F. H., \& Tomasello, M. (2012). Young children enforce social norms. Current Directions in Psychological Science, 21, 232-236. doi:10.1177/0963721412448659

Schwier, C., Van Maanen, C., Carpenter, M., \& Tomasello, M. (2006). Rational imitation in 12-monthold infants. Infancy, 10, 303-311. doi:10.1207/s15327078in1003_6

Shneidman, L., Todd, R., \& Woodward, A. (2014). Why do child-directed interactions support imitative learning in young children? PLoS One, 9, e110891. doi:10.1371/journal.pone.0110891

Smetana, J. G., Kochanska, G., \& Chuang, S. (2000). Mothers' conceptions of everyday rules for young toddlers: A longitudinal investigation. Merrill-Palmer Quarterly, 46, 391-416.

Tomasello, M. (2016). A natural history of human morality. Cambridge, MA: Harvard University Press.

Tomasello, M., \& Vaish, A. (2013). Origins of human cooperation and morality. Annual Review of Psychology, 64, 231-255. doi:10.1146/annurev-psych-113011-143812

Tuncgenc, B., Hohenberger, A., \& Rakoczy, H. (2015). Early understanding of normativity and freedom to act in Turkish toddlers. Journal of Cognition and Development, 16(1), 44-54. doi:10.1080/15248372.2013.815622

Vaish, A., Carpenter, M., \& Tomasello, M. (2009). Sympathy through affective perspective taking and its relation to prosocial behavior in toddlers. Developmental Psychology, 45, 534-543. doi: $10.1037 / \mathrm{a} 0014322$

Vaish, A., Missana, M., \& Tomasello, M. (2011). Three-year-old children intervene in third-party moral transgressions. British Journal of Developmental Psychology, 29(1), 124-130. doi: $10.1348 / 026151010 \times 532888$

Wyman, E., Rakoczy, H., \& Tomasello, M. (2009). Normativity and context in young children's pretend play. Cognitive Development, 24(2), 146-155. doi:10.1016/j.cogdev.2009.01.003

Received 23 November 2015; revised version received I 7 June 2016

\section{Appendix: Coding categories for enforcement}

\begin{tabular}{|c|c|c|}
\hline Type of enforcement & Description & Examples \\
\hline Imperative protest & $\begin{array}{l}\text { Objecting to the puppet's action using } \\
\text { imperative language }\end{array}$ & $\begin{array}{l}\text { Don't do that! } \\
\text { Put it through! } \\
\text { This way! }\end{array}$ \\
\hline \multirow[t]{10}{*}{ Normative protest } & $\begin{array}{l}\text { Objecting to the puppet's action using } \\
\text { either of the following: }\end{array}$ & \\
\hline & Normative vocabulary: & That's wrong! \\
\hline & must, have to, should, ought, right, & You must do it like this! \\
\hline & correct, wrong & You should put it through! \\
\hline & Normative phrases: & \\
\hline & It goes like this. & \\
\hline & That's not how it goes. & \\
\hline & This is how one does (not do) it. & \\
\hline & Normative object references: & The stick goes there! \\
\hline & belong, go & This doesn't belong in there \\
\hline
\end{tabular}

